\title{
Urbanización de áreas inundables, mediación técnica y riesgo de desastre: una mirada crítica sobre sus relaciones ${ }^{1}$
}

\author{
Diego Martín Ríos ${ }^{2}$
}

\begin{abstract}
RESUMEN
Las reflexiones aquí presentadas procuran enriquecer las discusiones teóricas sobre desastres y riesgos de desastre efectuadas por las Ciencias Humanas y Sociales (en particular por la Geografía) hasta el momento. Ello se comete a partir de una mirada crítica sobre las relaciones que se establecen entre urbanización de áreas inundables, mediación técnica y riesgo de desastre, en términos de lo que se ha llamado de "producción de espacio de riesgo de desastre". En el trabajo se destaca el lugar que ocupa la mediación técnica en la producción de ese tipo de espacios en sus dimensiones económica, política e ideológica. En particular se analiza la singularidad que adquieren las técnicas hidráulicas en la urbanización de áreas inundables tanto en términos de solución única ante las inundaciones, de estrategia de valorización urbana de esas áreas, como de respuesta que termina amplificando al propio riesgo de desastre.
\end{abstract}

Palabras clave: Urbanización de áreas inundables, mediación técnica, riesgo de desastre.

\begin{abstract}
The following argument attempts to enrich the theoretical discussion on disasters and disaster risks that have taken place in the Human and Social Sciences (particularly in Geography) until now. This is done through a critical review of the relations normally established between urbanization in flood-prone areas, technical mediation and disaster risk, in terms of what we have called "disaster-risk space production". In this work we highlight the role of technical mediations in the production of these areas, considering the economic, political, and ideological dimensions involved in the process. Specifically, we analyze the singularity of "hydraulic techniques" in the urbanization of flood-prone areas, as the 'unique solution' to flood problems, as a strategy to increase the value of those areas, and as a response that ultimately amplifies disaster risk.
\end{abstract}

Key words: Urbanization of flood-prone areas, technical mediation, disaster risk.

\footnotetext{
Este trabajo se basa parcialmente en el marco teórico-conceptual que el autor desarrolló para su tesis doctoral. Artículo recibido el 1 de octubre de 2010 y aceptado el 13 de octubre de 2010 .
}

2 Instituto de Geografía, Universidad de Buenos Aires (Argentina). E-mail: diegorios@conicet.gov.ar 
Prácticamente a diario los medios de comunicación nos informan de la ocurrencia de nuevos desastres "naturales" en algún lugar del mundo y de la cantidad de personas y de bienes por ellos afectados. Desde hace varias décadas las Ciencias Físicas y aplicadas han avanzando en el conocimiento científico sobre las propiedades de los fenómenos físicos extremos desencadenantes, confundiéndolos muchas veces con el desastre mismo. Por su parte, las Ciencias Humanas y Sociales han desarrollando, más recientemente, perspectivas propias sobre la comprensión de los desastres centrando su análisis en las condiciones de vulnerabilidad social que anteceden a estos eventos. A pesar de ello, aún son escasos los trabajos en los que se presentan marcos teórico-conceptuales que ponen de relieve la profunda naturaleza social de los llamados desastres "naturales" y la complejidad de aspectos que participan de su producción; esto es, el riesgo de desastre en tanto potencialidad de concreción/materialización del desastre.

Este artículo pretende reflexionar críticamente sobre la dinámica que adquiere la articulación entre los procesos de urbanización, mediación técnica y riesgo de desastre, especialmente para el caso de las áreas inundables, pero que a pesar de su singularidad puede pensarse para áreas donde tienen lugar otros fenómenos físicos extremos. Para ello se presentan las discusiones sobre desastres y riesgo de desastre y sobre algunos aportes significativos que permiten reparar, principalmente desde la Geografía, en lo que hemos llamado de "producción de espacios de riesgo de desastre". Luego, se prosigue en torno a las discusiones sobre el par riesgo de desastre-ciudad, dada la importancia que han adquirido los núcleos urbanos y sus sucesivas transformaciones sociales y espaciales en tanto partícipes centrales de la conformación de riesgos y de desastres en todo el mundo. Seguidamente, se resalta el lugar destacado que ocupa la mediación técnica en la producción de espacios de riesgo de desastre, señalando las especificidades que adquieren las técnicas "hidráulicas" en la urbanización de áreas inundables tanto en términos de "solución única" ante las inundaciones, de estrategia de valorización urbana de esas áreas, como de respuesta que termina amplificando al propio riesgo de desastre. Por último, se presentan las consideraciones finales.

\section{Del desastre a la producción de riesgo de desastre}

Los desastres y sus relatos forman parte de la historia de la Humanidad. El gran Diluvio del Génesis bíblico, la peste negra en Europa medieval (1350) o la explosión atómica de Chernobyl (1986), son solo algunos ejemplos de ello. Sin embargo, estos fenómenos comenzaron a ser objeto de tratamiento científico no hace mucho tiempo. Las Ciencias Físicas y aplicadas (las ingenierías), fueron quienes lideraron los estudios científicos, concibiendo a los fenómenos físicos desencadenantes (naturales, tecnoindustriales, etc.) como los causales de los desastres. Desde esta concepción, la naturaleza de una inundación, un terremoto o una explosión de una central térmica, constituyen "el peligro" y el daño se relaciona a la magnitud, frecuencia y otras dimensiones de esos procesos físicos extremos, concediéndole a la sociedad un lugar secundario asociado principalmente a su capacidad de respuesta.

Hewitt (1983) ha llamado a esta concepción del desastre como la "visión dominante" y Lavell (1996a) como el "paradigma fisicalista", puesto que se centra no solo en el conocimiento del fenómeno físico de origen natural o tecnoindustrial, sino que las respuestas pasan, mayoritariamente, por medidas de claro corte ingenieril o técnico. Así, los desastres son concebidos como una anormalidad, una disrupción de un estado estable, como eventos impredecibles, inmanejables e inevitables (Hewitt, 1983). En consecuencia, el carácter de "producto" que se le otorga al desastre hace que sea solo atendible cuando estos ocurren.

Los aportes de las Ciencias Humanas y Sociales en relación al conocimiento en torno a los desastres vienen recorriendo un extenso y serpenteante camino, cuyos comienzos, de manera más metódica y constante, se inician en la década de 1940, en los Estados Unidos. Después de las grandes inundaciones ocurridas en la cuenca del Mississippi en 1927, el gobierno de Roosevelt promovió un proyecto faraónico de inversión de importantes 
obras hidráulicas, bajo el marco de desarroIlo de políticas de corte keynesiano. Luego de varios años de avanzado ese proyecto, el geógrafo Gilbert White (quien participó como asesor) advirtió que la solución a estos problemas no consistía en la construcción de mayor cantidad de obras hidráulicas, tal como se venía haciendo, sino en analizar las relaciones entre la sociedad y esas naturalezas particulares (Saurí, 2006).

White, formado en las teorías funcionalistas de la escuela de la ecología humana de la Universidad de Chicago, proponía analizar los extremos naturales, especialmente las grandes inundaciones, como formas complejas en que interactuaban la sociedad y la naturaleza, desde el punto de vista del ajuste humano al ambiente. Según Calderón (2001), con la escuela de White (y sus colaboradores Burton y Kates) se planteó por primera vez la participación social en los desastres; no obstante ello, ese tratamiento continuó estando orientado al conocimiento de las sietes variables asociadas con los fenómenos desencadenantes del desastre, estas son: magnitud y velocidad de ataque; frecuencia y duración; espacialización temporal; área de extensión y dispersión espacial. Marandola \& Hogan (2004) sostienen que a pesar que esta propuesta centraba su análisis en la antigua relación sociedad-naturaleza, el mayor desarrollo dentro de la disciplina quedó circunscrito al conocimiento de las propiedades del fenómeno natural. Es así como los geógrafos físicos fueron los que tomaron la posta en las investigaciones en torno a los desastres, por lo que sus análisis poseían una fuerte componente físico más allá de los intentos de integrar los elementos físicos y sociales.

Hacia fines de la década de 1970 el punto de partida de la ecología humana sobre los desastres es enriquecido con el desarrollo de la escuela de la economía política, la cual va a centrar su análisis en la vulnerabilidad social. Esta escuela -enmarcada en las corrientes neomarxistas que se desarrollan en esos tiempos en distintas disciplinas sociales, como la Geografía radical- surge de la Disaster Research Unit de la Universidad de Bradford, Inglaterra, liderada por autores tales como O'Keefe, Watts, Westgate, Wisner, entre otros. Estos autores comenzaron a interiorizarse en el tema de los desastres a partir de estudios de caso sobre sequías y hambrunas acontecidas en zonas rurales del norte de África a comienzos de los setenta ( $\mathrm{O}^{\prime}$ Keefe et al., 1976; Westgate \& O'Keefe, 1976; Watts, 1983; Lavell, 2004).

Los desastres, según la escuela de la economía política, son el resultado de procesos socioeconómicos que crean, a distintas escalas, condiciones de existencia humana insostenibles ante los eventos extremos y que se diferencian en términos de clase, raza, género, edad, etc. Es por ello que para estos autores el concepto central no es el desastre, sino el riesgo de desastre en tanto condición de esa potencialidad negativa contenida en los desastres. Para Wisner (2002), en general la propia 'Humanidad' y 'Sociedad' no están 'en riesgo', sino que están en riesgo determinados grupos sociales con características específicas. Las formas en que se fueron estructurando históricamente las condiciones de vulnerabilidad ante riesgos de desastre, más allá de las características que adopta el desastre mismo (aspecto central de los enfoques precedentes), constituyen el objeto de análisis más importante para esta perspectiva. Según los especialistas en la temática (Lavell, 2004), la economía política de los desastres sentó las bases de lo que con posterioridad se denominaría como la Escuela de la Vulnerabilidad, con gran auge en las décadas siguientes.

Ya hacia fines de la década de 1990 e inicios de este nuevo siglo, se produjo un consenso en cuanto a la terminología común sobre riesgo de desastre, tanto por parte de los investigadores inscriptos dentro de las visiones alternativas como por parte de organismos de cooperación internacional (por ejemplo: la Organización de las Naciones Unidas para la Ayuda en Desastres). Según este acuerdo el riesgo de desastre está formado por dos dimensiones: la amenaza (o peligro) y la vulnerabilidad. Ello es expresado bajo la fórmula $\mathrm{R}=\mathrm{A} \times \mathrm{V}$ (donde $\mathrm{R}=$ Riesgo, $\mathrm{A}=$ Amenaza y $\mathrm{V}=$ Vulnerabilidad).

Siguiendo a Lavell (1996b), las amenazas (o peligros) constituyen eventos físicos latentes, esto es, probabilidades de ocurrencia de eventos físicos dañinos. Las amenazas son clasificadas según el origen de las mismas en: naturales, socionaturales y antropogénicas (a 
las que otros autores mencionan de tecnológicas o tecnoindustriales).

La vulnerabilidad, por su parte, refiere a las condiciones sociales, económicas, culturales, políticas, institucionales, etc., de una sociedad, que existen previamente a la ocurrencia de un desastre. Estas características definen la capacidad de la sociedad para anticipar, resistir y recuperarse de los efectos de una amenaza (Blaikie et al., 1994). Desde esta perspectiva, esta capacidad se presenta de manera diferente de acuerdo a las heterogeneidades de las sociedades implicadas. En otras palabras, situaciones sociales y de desarrollo económico diversas explican cómo peligros similares pueden tener efectos disímiles en diferentes países y sociedades.

Para consolidar la interpretación teórica de la economía política del riesgo, Mansilla (2000) considera que debe avanzarse en la caracterización y el análisis de la base material de los riesgos de desastre, es decir, en la construcción histórica de las condiciones de riesgo, previa al desastre como acontecimiento. Cuando la base material que da lugar a la ocurrencia de estos eventos no forma parte de la explicación, el desastre es solo concebido como resultado, como situaciones de crisis, disrupción, muerte y pérdida de bienes materiales (concepción de la visión aún predominante). Por el contrario, el enfoque neomarxista de la economía política aporta elementos suficientes para esclarecer la base material y objetiva de los desastres, puesto que "a partir de la concepción material del mundo, la explicación del impacto de fenómenos naturales sobre la sociedad pierde su carácter de 'divino' y se convierte en resultado de hechos humanos" (Mansilla, 1996: 61).

En el análisis materialista de los riesgos de desastre, afirma Mansilla (2000), es necesario realizar una distinción entre los actores generadores y actores reguladores del riesgo. El riesgo de desastre se construye y agudiza respondiendo a intereses individuales y de carácter privado, al tiempo que su resultado -el desastre- colectiviza sus efectos. Los actores privados y los agentes individuales toman decisiones que contribuyen a la generación de riesgos de desastre, por lo que se indica una suerte de privatización en ese proceso de construcción, mientras que el Estado se ha transformado, principalmente, en el actor regulador, a través del manejo de los desastres, quedando entonces la responsabilidad ante las consecuencias en la órbita pública. Asimismo, se señala en varios de estos trabajos la participación del Estado como actor generador de riesgos de desastre, permitiendo, por ejemplo, los asentamientos irregulares o la construcción de obras de infraestructura que amplifican el potencial dañino de los fenómenos físicos extremos.

Quien logra alcanzar un aporte teórico sugestivo y que termina superando la concepción todavía arraigada en la temática (tanto en la Geografía como en las otras disciplinas) en la que la naturaleza (amenaza o peligro) se encuentra separada de la sociedad (vulnerabilidad), es la propuesta realizada por la geógrafa mexicana Georgina Calderón. En su tesis doctoral, en formato de libro, Calderón (2001) recupera los avances realizados por la economía política de los riesgos de desastre, especialmente de las escuelas anglosajonas, y prosigue en la construcción de esa perspectiva, resaltando que para entender a los desastres y los riesgos de desastre deben analizarse las formas en que nuestras sociedades producen sus espacios $y$, especialmente, las particularidades que ese proceso adopta en las sociedades capitalistas.

La producción de espacio en las sociedades capitalistas se caracteriza, como sostiene Calderón (2001), por su desarrollo desigual. Esa diferenciación espacial tiene como principio la división del trabajo. Esto origina que la población tenga diferente acceso a los recursos generados por la misma sociedad; y esas determinaciones son las que gestan la vulnerabilidad de los miembros de una sociedad. De esta manera, las poblaciones se encuentran en riesgo porque ha habido una producción de espacios, que de acuerdo con las características socioeconómicas de la población que los crea, se convierten en riesgosos, y ello se expresa desigualmente; esto es, el riesgo no es para todos por igual, sino que es más decisivo para determinados grupos que para otros.

En este sentido, el riesgo de desastre se produce "de acuerdo con la forma de apropiación diferencial de la naturaleza, según las condiciones socioeconómicas de quien 
se apropia de ella, no solo de las características físicas de la misma" (Calderón, 2001: 73). Las concepciones que dividen al peligro de la vulnerabilidad, esto es, a la naturaleza de la sociedad, reducen, según Watts "a los hombres a objetos, en donde pierden irremediablemente el papel de sujetos y agentes históricos, les quitan por tanto la categoría de seres productores conscientes, activos e intencionados de las relaciones sociales y condiciones materiales" (Calderón, 2001: 76). Es por ello que preguntarse por qué, desde cuándo, quién y cómo la sociedad, diferenciada en grupos, construye espacios que los vuelven riesgosos, constituyen -para esta autora- algunos de los interrogantes centrales para poder comprender estas problemáticas complejas. En esta misma línea de argumentación, Kelman (2007) sostiene que los fenómenos naturales se tornan peligrosos para las sociedades debido a las decisiones realizadas por estas, es decir, que la naturaleza de por sí no produce peligros, sino que las sociedades deciden si esos fenómenos pueden ser peligrosos para ellas o no, en tanto hayan construido situaciones de riesgo, que tienen una clara expresión espacial, por lo que podemos hablar de espacios de riesgo de desastre y de su producción.

\section{Riesgo de desastre y ciudad}

Tanto los estudios sobre riesgo de desastre como aquellos vinculados propiamente con la ciudad han constituido dos campos del saber escindidos por mucho tiempo. Como se ha mencionado anteriormente, los estudios sobre desastres asociados a la visión dominante, estuvieron dirigidos al avance del conocimiento de los fenómenos físicos en tanto generadores de los desastres, por lo que las causalidades sociales de base urbana han sido dejadas de lado en la explicación. Recién a mediados del siglo XX se emprendió la realización de estudios que, pertenecientes a la visión alternativa liderada por las Ciencias Humanas y Sociales, fueron incorporando las temáticas urbanas vinculadas con los desastres y los riesgos de desastre.

En las últimas décadas, se observa un notable avance en la realización de trabajos que procuran abordar las articulaciones entre riesgo de desastre y ciudad. Ello se enmarca en la importancia que viene adquiriendo la población urbana a nivel mundial, como también el papel destacado que están alcanzando las ciudades en cuanto a la concentración del poder económico y político en la etapa actual de la globalización capitalista.

Varios autores vienen señalando a los procesos de urbanización como las principales causales de situaciones de riesgo de desastre en el mundo, en tanto que, por un lado, la forma en que estos se desenvuelven alteran las dinámicas naturales (y con ella los peligros) y, por otro, la influencia que estos ejercen es claramente significativa en la generación de condiciones de vulnerabilidad social, como también en las medidas que se puedan tomar para mitigar y prevenir riesgos (Mitchell, 1999). La degradación ambiental urbana, la cual no solo amplifica los peligros sino que adquiere fuerte incidencia en el incremento de las vulnerabilidades, es otra de las causas de la conformación de riesgo de desastre en los ámbitos urbanos (Herzer y Gurevich, 1996; Lavell, 1996b). Estos procesos urbanos, en un contexto de mayor velocidad e intensidad en el que se están produciendo cambios, lleva a autores tales como Mansilla (2000) a considerar a las ciudades como el nuevo escenario del riesgo.

Para entender el riesgo de desastre en los ámbitos urbanos se deben comprender las formas en que se producen las ciudades. Las condiciones naturales en el proceso de urbanización son incorporadas de acuerdo con las lógicas sociales del modo de producción imperante en cada momento histórico. Así, las diferencias en las condiciones naturales que forman parte de las condiciones de sitio, son integradas al proceso urbano de manera desigual, especialmente con el desarrollo del capitalismo que, a partir del fenómeno de la industrialización, hace explotar a las ciudades a tasas de crecimiento nunca antes vistas. Los valles de inundación, las laderas inestables o de volcanes activos constituyen áreas donde ocurren fenómenos naturales extremos que pueden derivar en peligros, las cuales también forman parte de los recursos y sustentos materiales, por lo que son distribuidas socialmente de manera desigual. Por lo general, por lo menos para el ámbito latinoamericano, son los enormes contingentes de migrantes internos en condición de pobreza los que ocupan esas áreas debido a sus esca- 
sas posibilidades de acceso al mercado legal de tierras (Calderón, 2001).

Una de las perspectivas que más ha incursionado en las últimas décadas en la explicación de la dinámica urbana y de la conformación de los riesgos de desastre, es la economía política. Veamos aquí algunos ejemplos.

En el contexto latinoamericano son varios los trabajos que articulan el par riesgo de desastre-ciudad a través del análisis de casos de estudio, sin embargo, son raros aquellos que junto al trabajo empírico también aportan una estructura teórico-conceptual para la interpretación de las mutuas influencias entre estas dos dimensiones. Dentro de este último grupo se encuentran, por ejemplo, los trabajos de las mexicanas Elizabeth Mansilla (1996; 2000) y Georgina Calderón (2001), con clara referencia a la escuela de la economía política de los riesgos de desastre, cuyo objeto, en base al método del materialismo histórico, consistió en develar la base material en la conformación de estos complejos procesos a lo largo del tiempo.

En su tesis de doctorado, intitulada Riesgo y ciudad, Mansilla (2000) aporta una enriquecedora interpretación teórico-empírica sobre el proceso de construcción de riesgo de desastre, tomando el caso de la Ciudad de México desde el momento de la Conquista y Colonización hasta fines del siglo XX. En su estudio focaliza especialmente el manejo hidráulico que se ha dado en esa cuenca, debido a que el agua ha sido el elemento determinante en la forma de socialización de la naturaleza a lo largo de toda la historia de esa ciudad. El sistema hidráulico que opera en la actualidad, tiene su origen en las necesidades de reproducción del capital y en la importancia que en ese sentido dicha ciudad fue adquiriendo para México. Ello se ha complejizado en una urbe que ha crecido desmesuradamente, pasando de poco más tres millones de habitantes en 1950 a más de dieciséis millones en 2000. Ese proceso estuvo liderado (y continúa siéndolo) por migrantes internos pobres que terminan habitando asentamientos precarios (muchas veces ilegales), sin servicios básicos, localizados, generalmente, en sitios desfavorables ambientalmente. Para esta autora, todo indica que la más populosa de las ciudades latinoamericanas se dirige hacia un desastre anunciado de graves consecuencias, en el que es sabido de antemano quienes serán los principales afectados.

Calderón (2001), por su parte, aborda la construcción de riesgo de desastre para el caso de la ciudad-puerto de Manzanillo, a partir de la diferenciación espacial de la vulnerabilidad, teniendo como basamento el desarrollo del modo de producción capitalista en la zona. Esta autora destaca que los procesos de urbanización de esa ciudad están vinculados, en distintos momentos históricos, con el desarrollo de las industrias petroquímicas y de las actividades de servicios tales como el transporte y el turismo. El desenvolvimiento de cada una de estas actividades estimuló la radicación de migrantes internos en Manzanillo en busca de mejores condiciones de vida. Dadas las situaciones socioeconómicas de privación y la dinámica del mercado de suelos en esa ciudad, esos grupos terminaron ocupando áreas sujetas a fenómenos físicos extremos como son las laderas inestables. La forma en que fueron incorporadas esas características del medio por parte de esos grupos demuestra la configuración de la vulnerabilidad diferencial como corolario de ese proceso de urbanización dentro de la lógica del modo de producción capitalista.

Ya en el contexto norteamericano, Mike Davis escribió algunos trabajos (Davis, 1995; Davis, 1999) en los que efectúa un pormenorizado análisis sobre la forma en que el capitalismo ha construido la ciudad de Los Ángeles como una zona de desastres potenciales. A pesar que este autor concluye en dichos trabajos que la urbanización de esa ciudad ha acontecido en uno de los momentos más benignos en términos climáticos y sismológicos de su historia natural, esa metrópolis del sur californiano se ha transformado en una mezcla explosiva de peligros naturales y contradicciones sociales (Davis, 1999).

En su libro Ecology of fear, Davis (1999) analiza cómo los distintos tipos de fenómenos naturales (incendios, tornados, inundaciones, terremotos, animales y plagas, entre otros) propios de ese ambiente, son convertidos en riesgos de desastre por el accionar social. El autor destaca las acciones del Estado y de los actores económicos privados vincu- 
lados al Real Estate (bienes raíces), como los actores sociales con mayor responsabilidad en la generación de esos fenómenos; acciones que favorecen la construcción de una "amnesia del desastre" en esas sociedades. Son sugerentes sus análisis de la ocurrencia de incendios provocados por la forma en que se produce el espacio residencial para las elites. Este es el caso de la zona de Malibú, que casi todos los veranos se ve afectada por incendios en los que la propia forestación implantada y los bienes inmuebles allí construidos (con abundante presencia de maderas), sirven de combustible (a veces con saldos trágicos), olvidándose, convenientemente, que Los Ángeles se encuentra en una zona de clima mediterráneo donde el fuego constituye uno de los principales pulsos de ajuste natural.

Otra interesante indagación sobre el par riesgo de desastre-ciudad es la que Ileva adelante el historiador Ted Steinberg (2001) en su artículo The Secret History of Natural Disaster (resultado de su tesis doctoral). En esa publicación se detalla el papel significativo que ha tenido la acelerada expansión de la urbanización capitalista en los Estados Unidos, sobre todo hacia fines del siglo XIX $y$, especialmente, con la gran suburbanización de las décadas de 1950 y 1960 luego de la posguerra, junto a la gestión ineficiente por parte del Estado, en la construcción de los desastres naturales. Este autor retoma la idea de la "amnesia del desastre" de Davis, para entender la lógica con la que los actores con mayor poder incorporan áreas potencialmente peligrosas (laderas de montañas y pedemontes - para el caso del sur de California- o pantanos y áreas costeras -para el caso del sur de la Florida-) al proceso de urbanización, especialmente, en períodos donde los desastres no acontecen debido a la falta de eventos detonantes significativos. Este autor norteamericano resalta que la ausencia de sismos en el sur de California entre las décadas de 1950 y 1960 coincide con la construcción de más de 60.000 viviendas en sitios inapropiados o el caso de la ausencia del paso de huracanes en el sur de la Florida entre las décadas de 1970 y 1990, permitiendo la expansión de urbanizaciones cerradas (gatted communities) y de complejos hoteleros en áreas inundables y costeras de ese turístico estado.
Los casos tratados por Davis y Steinberg permiten despegarnos del vínculo tradicional (o por lo menos mucho más analizado) en el que se relaciona a los desastres solo con los grupos más desprotegidos. Estos autores muestran, en cambio, como los grupos de mayores ingresos no están exentos de esos acontecimientos, aunque cuenten con recursos suficientes para sobreponerse y elaboren estrategias exclusivas para mitigar los impactos, en tanto condiciones de menor vulnerabilidad social ${ }^{3}$.

\section{Técnica y producción de espacio de riesgo de desastre}

En el proceso de producción de espacio en áreas proclives a la ocurrencia de fenómenos físicos extremos, es esencial comprender el papel de las técnicas para disminuir los efectos adversos que se puedan derivar de esa situación. En las sociedades capitalistas el acceso a esas técnicas es claramente desigual. No obstante ello, pocos se preguntan, tal como sostiene Calderón (2001), quiénes son los que tienen la posibilidad real de acceder a esos "adelantos" técnicos, es decir, qué proporción de la sociedad puede alcanzar al sistema científico-técnico actual, y cómo los avances en las técnicas se manifiestan también en el espacio, diferenciándolo.

Muchas veces pareciera que la técnica no tuviera un lugar destacado en la producción de espacio en general y de riesgo de desastre en particular. Uno de los geógrafos que más ha avanzado en esa dimensión ha sido Milton Santos. Para este autor, la forma en que las sociedades producen sus espacios no puede entendérsela sin comprender el papel que adquiere la técnica. El incesante proceso de

\footnotetext{
3 Quien ha analizado las estrategias de evacuación y su mercantilización en los Estados Unidos es Noemí Klein (2007). Según esta autora canadiense, distintas empresas de seguridad brindan servicios a la franja más selecta de los grupos con mayor poder económico de ese país, tal es el caso de servicios aéreos para trasladar de manera privada y efectiva a los propietarios de las más suntuosas viviendas de la Florida a hoteles cinco estrellas antes de que el huracán toque tierra, o del servicio de sistema de spray antifuego ofrecido a los magnates de las mansiones del sur de California para evitar que sus bienes sean consumidos por las llamas.
} 
renovación en las técnicas, precisamente, va a permitir explicar, no en su totalidad pero sí como parte importante de esa complejidad, las permanentes modificaciones y transformaciones que se van configurando en el espacio en el devenir histórico (Santos, 1994).

La historia del hombre sobre la Tierra es la historia de una ruptura progresiva entre el hombre y la naturaleza, proceso que se acelera cuando el hombre se descubre como individuo e inicia una mecanización del planeta, armándose de nuevos instrumentos técnicos para dominarlo (Santos, 1994). La sustitución de un medio natural por un medio cada vez más artificial, cada vez más instrumentalizado, con sus particularidades en cada uno de los lugares de la Orbe, da cuenta de este camino que la sociedad humana le impone al Planeta (Santos, 2000).

Para el filósofo Jürgen Habermas (1985), la propia racionalidad técnica debe ser pensada como ideología, puesto que “... no es que determinados fines e intereses de dominio solo se advengan a la técnica a posteriori y desde fuera, sino que entran ya en la construcción del mismo aparato técnico. La técnica es en cada caso un proyecto históricosocial; en él se proyecta lo que una sociedad y los intereses en ella dominantes tienen el propósito de hacer con los hombres y con las cosas" (Habermas, 1985: 55).

En las sociedades modernas, la ciencia y la técnica han proporcionando "tanto los conceptos puros como los instrumentos para una dominación cada vez más efectiva del hombre sobre el hombre a través de la dominación de la naturaleza" (Habermas, 1985: 58). Para Meindl et al. (2002), la ciencia y la técnica se encuentran entre las más contundentes estrategias para alcanzar esa dominación, así lo demuestra el lugar que se le ha otorgado a estas y que se expresa en la importancia que adquiere el discurso científico-técnico en nuestras sociedades. A partir de la Modernidad, la ciencia y la técnica han sido presentadas como verdades objetivas, moralmente neutras y separadas de los intereses sociales. En realidad, tal como plantea Noel Castree, no existe una forma sencilla de escindir las observaciones objetivas de la ciencia y la técnica, de las influencias sociales y de los intereses políticos y económicos
(Meindl et al., 2002). El Estado y los actores económicos privados se encuentran involucrados en la producción y legitimación del conocimiento científico-técnico, el cual está fuertemente condicionado por las consideraciones políticas y económicas, de allí que se utiliza la frase la ciencia del mandato para referirse a esta cuestión.

Por ejemplo, Meindl et al. (2002) mencionan que los cuerpos técnicos (especialmente los ingenieros) obtienen legitimidad por el conocimiento científico-técnico que retienen, por su posición privilegiada como los cuerpos de elite política dentro del aparato del Estado, y por constituirse, también, en los consejeros de los intereses de los actores económicos privados. Los discursos elaborados por estos grupos, asimismo, asumen una autoridad intelectual que ayuda a legitimar la aplicación de técnicas tanto social como políticamente; esto es, un discurso que permite la conversión de ideas técnicas en hechos sociales aceptados y que son utilizados en la arena pública. Es por ello que el conocimiento desarrollado por estos grupos (ingenieros y otros cientistas aplicados) participa de manera significativa en la forma en que el resto de la sociedad entiende las condiciones físicas del espacio y la "necesidad" de su transformación a partir del continuo avance del desarrollo técnico.

Para Santos (1994), entre otros autores, el estudio de las técnicas, entonces, debe superar ampliamente el dato propiamente técnico y exigir una incursión más profunda en el área de las propias relaciones sociales de producción. Las técnicas, según O'Connor (2001), tienen distintos significados y funciones sociales, económicas, políticas e ideológicas, que pueden variar según los contextos espacio-temporales. En el modo de producción capitalista, la elección de determinada técnica no se realiza principalmente en función a los principios ecológicos, sino teniendo en consideración los costos y las ventas.

Toda intervención humana sobre la naturaleza conlleva un grado de transformación. Sin embargo, esa transformación ha adquirido niveles alarmantes asociados al avance del desarrollo científico-técnico sumamente acelerado en los últimos tiempos. Mientras más rápido es el proceso de transformación, 
más corto es el tiempo que tienen los ecosistemas para adaptarse naturalmente a esos cambios, produciéndose una alteración de las condiciones naturales o una degradación del hábitat. Es evidente que este creciente nivel de transformación de la naturaleza se aleja cada vez más de su motivo original: satisfacer necesidades humanas, y se acerca progresivamente a las necesidades de reproducción del propio capital (Mansilla, 2000).

Generalmente, los análisis mayoritarios que se realizan en torno a las técnicas se reducen al grado de modernidad, efectividad, monto invertido, actividades económicas que involucran y estimulan, etcétera, dejando de lado, tal como indican Pérez Picazo y Lemeunier (1990), las conexiones profundas que las técnicas poseen con los factores productivos y las estructuras socioeconómicas y de poder; aspectos que son sumamente necesarios de abordar para lograr comprender y explicar las razones de la existencia de unas o de otras en determinado espacio/tiempo.

\section{El papel de las técnicas "hidráulicas" en la urbanización de áreas inundables}

Las áreas inundables son definidas por los especialistas en hidrología, geomorfología y topografía como áreas planas ubicadas junto a un curso de agua, las cuales son cubiertas por el agua durante una crecida. También a ellas se las conoce como llanura o valle de inundación (OEA, 1993). Como se puede observar en esta definición se introducen los conceptos de crecida e inundación, los cuales no remiten a lo mismo. Según Paoli y Giacosa, las crecidas forman parte del régimen normal de un curso de agua y constituyen la respuesta hídrica frente al exceso en los aportes de aguas originadas por lluvias excesivas o deshielos (González, 2009). Por su parte, una inundación, ocurre cuando una crecida supera la capacidad de carga de los ríos y de absorción de los suelos, y afecta la regulación del sistema de defensa que establece la sociedad, produciendo daños en ella.

Este primer acercamiento en el que son destacadas las condiciones geográficas de sitio y las características físico-naturales de esos fenómenos es complementado por las aproximaciones que realizan disciplinas tales como la Geografía Humana, que complejizan esa definición al incorporar las características socialmente producidas en esos espacios, es decir, que esas condiciones constituyen la base material de las relaciones sociales que se construyen históricamente, cambiando su configuración original a través de sucesivas transformaciones. En este sentido, a partir de este tipo de interpretaciones se pretende avanzar sobre el análisis tradicional que se realiza sobre las inundaciones urbanas, el cual se limita, a menudo, a la cantidad de agua caída y a la alteración del ciclo del agua que se produce en los espacios urbanizados, en cuanto al desequilibrio en la ecuación infiltración-escurrimiento (disminuyendo la primera y aumentando la segunda) (González, 2009).

La urbanización de áreas inundables ofrece una dinámica singular en los procesos de producción de riesgos de desastre en los ámbitos urbanos. La mayoría de las ciudades del mundo se encuentran situadas a la vera de ríos u otros cuerpos de agua. Este vínculo nace con las primeras ciudades en tiempos de la Antigüedad (3.000 años a. C.), tal como lo atestiguan los restos de la ciudad de Ur, a la vera del río Éufrates en el sur de la Mesopotamia (Nel-Lo y Muñoz, 2004). Ello demuestra la importancia que ha tenido el agua como condición necesaria para la reproducción de la vida a lo largo de la historia humana. Este tipo de localización no solo estuvo motivada para satisfacer las necesidades de abastecimiento de agua, sino, también, las de aprovisionamiento de alimentos, de transporte y comunicación, de depósito de desechos, de lugar para la expansión urbana, de recreación y esparcimiento, entre otras.

La elección por este tipo de localizaciones implicó, asimismo, que esas sociedades tuvieran que convivir con fenómenos potencialmente dañinos como son las inundaciones que se manifiestan de tanto en tanto, de acuerdo a los ciclos hidroclimáticos. Sin embargo, los beneficios que esta localización acarrea a las sociedades allí establecidas superan por mucho las pérdidas de vidas o de bienes que se generan cuando se produce una inundación (Roswell, 1997). La relación entre ciudad y áreas inundables guarda un 
carácter complejo y dinámico, fruto de la combinación, históricamente cambiante, de recursos y oportunidades aportados por los cursos de agua, y de riesgos (Moral, 1997), una vez que las sociedades construyen espacios en sus áreas de afectación, dependientes de los niveles de vulnerabilidad social alcanzados.

También debe destacarse que las ciudades que se encuentran en áreas inundables no son enteramente inundables, sino que existen algunas zonas de ellas más inundables que otras, de acuerdo a las condiciones naturales heterogéneas propias de cada sitio. Esas diferencias físico-naturales, al ser incorporadas en las relaciones sociales de producción, de las que forman parte el proceso producción de espacio urbano, terminan constituyendo diferencias sociales, las cuales se expresan espacialmente en la ciudad (Cutter, 2006). Esa diferenciación espacial establece que las zonas más inundables sean ocupadas, preferentemente, por los grupos de menores ingresos, de acuerdo a las pautas que establece el mercado de tierras en cada ciudad.

Nora Clichevsky (2003) es una de las autoras que más ha avanzado en la comprensión de la articulación entre mercado de tierras e inundaciones, especialmente, a partir de estudios de caso en algunas ciudades argentinas. Para esta autora es central destacar el papel que juega el Estado en las áreas inundables urbanas en tanto regulador de ese mercado, productor (de viviendas, por ejemplo) y constructor de defensas. Al mismo tiempo, también es necesario analizar los impactos que han tenido las inundaciones y las defensas en el mercado de tierra urbana, en términos de valorización/desvalorización y de enmascaramiento de estos fenómenos.

La incorporación urbana de áreas inundables a través de la utilización de diversas técnicas conserva una historia tan extensa como la propia historia de la urbanización. A grandes rasgos, las técnicas empleadas en la urbanización de áreas inundables procuraron solucionar dos tipos de problemas materiales diferentes: a) dominar los caudales de los cursos de agua y sus costas para disminuir los efectos adversos causado por las inundaciones y habilitar con ello los terrenos próximos para usos diversos, y b) mitigar los efectos dañinos que las aguas pueden ocasionar sobre edificaciones a través de la elevación del nivel de cota o la construcción de defensas en tierras inundables que ya han sido colonizadas.

Dentro de estos dos grandes grupos de técnicas, que aquí llamamos de técnicas "hidráulicas", es posible reconocer los siguientes tipos de obras. Entre las primeras, se encuentran las obras de dragado, canalización, rectificación o entubamiento de los cursos de agua. También se destacan los tablestacados para consolidar los bordes costeros y evitar o disminuir los procesos de erosión. Del segundo grupo de técnicas, por otro lado, sobresalen distintas obras constructivas de mitigación/defensa propias de las áreas inundables ya urbanizadas tales como: palafitos, diques, polderizaciones, terraplenes, pequeños rellenos para edificaciones individuales o grandes rellenos que ocupan hasta miles de hectáreas.

Las decisiones que se toman tanto para solucionar técnicamente el efecto negativo de las aguas como el lugar de emplazamiento de esa respuesta técnica, son dos aspectos centrales asociados con las relaciones de poder que se tejen en los procesos de urbanización de las áreas inundables. De la misma manera, también es interesante ahondar en la forma en que las propias técnicas "hidráulicas" se constituyen en amplificadoras de riesgo de desastre en las áreas inundables que se busca urbanizar, repercutiendo diferencialmente y reproduciendo las relaciones desiguales. Se verán algunos aspectos sobre estas tres dimensiones de este tipo de técnicas en el proceso de urbanización de áreas inundables.

\section{Las técnicas "hidráulicas" como "solución única"}

Para Erik Swyngedouw (1999) los cuerpos técnicos (especialmente ingenieros, cientistas aplicados y sus organizaciones colegiadas) cumplen un papel central en la opción de alternativas técnicas que se eligen para urbanizar las áreas inundables y/o mitigar los efectos negativos asociados a ellas. Esos cuerpos técnicos se constituyen en activos mediadores entre la sociedad y la naturaleza, y en partícipes directos de la transformación física de esa naturaleza inundable, produciendo 
naturalezas cada vez más tecnificadas. La misión de la ingeniería hidráulica (como máxima expresión de las técnicas "hidráulicas"), desde los inicios de la Modernidad, consistió en restaurar el equilibrio de los erráticos (y a veces invasores) cursos de agua, lo que requirió de una significativa empresa científica e ingenieril para entender y analizar las leyes de la naturaleza y para, con base a ese conocimiento, trabajar en su dominio técnico.

A lo largo de distintos momentos históricos la implementación de técnicas en la transformación urbana de áreas inundables ha sido realizada bajo el amparo de paradigmas científico-técnicos diversos, desde donde se elaboraron los discursos que le otorgaron sentido y argumentaron su legitimación. Entre la primera mitad del siglo XIX y comienzos del siglo $X X$, se fue estructurando, primero en Europa y luego en América, el paradigma higienista (y su correspondiente discurso) fundado en el punto de vista de las Ciencias Médicas y Naturales en relación con los procesos de modernización técnica que comenzaban a desarrollarse por esos tiempos, principalmente en los ámbitos urbanos. Este conocimiento científico-técnico, en el cual los médicos higienistas eran responsables del diagnóstico y los ingenieros de las intervenciones urbanas en nombre de la salubridad, consideraba a las tierras inundables cercanas a las ciudades como uno de los principales focos de miasmas y gérmenes que provocaban las pestes que asolaban a las poblaciones urbanas por aquellos tiempos (Seabra, 2008). Estos discursos contribuyeron a construir un imaginario cultural, todavía en alguna medida presente, en el que se equipara a las áreas inundables próximas a las ciudades con pantanos pestilentes e infectos, facilitando con ello su transformación técnica frente a las posibles resistencias sociales que puedan surgir para evitar esos procesos, dado la carga negativa transferida a esos ambientes (Bakker, 2005).

Desde ese entonces, bajo designios del higienismo comenzó un proceso técnico (que todavía no cesa, sino que se incrementa bajo formas específicas) en el que eran cegados, entubados y/o canalizados los ríos que atravesaban las ciudades, al igual que eran drenadas y/o rellenadas las áreas inundables próximas a ellas. Junto a ese proceso pro- piamente técnico, representado en las obras de infraestructura involucradas, fueron reglamentadas las prácticas urbanas de los habitantes originarios de esas zonas, con las cuales, también fueron vigilados sus cuerpos e, incluso, en varias ocasiones, terminaron siendo relocalizados o desplazados en nombre de la "salubridad" (Seabra, 2008; Silvestri, 2002). Así, las áreas inundables de las ciudades, junto a sus poblaciones pertenecientes, preferentemente, a grupos populares (muchas veces hacinados, pobres, desempleados, entre otros), se convirtieron, en aquellos tiempos, en objeto de intervención para el Estado. En consecuencia, comienzan a estructurase distintas oficinas técnicas especializadas que elaboraron políticas públicas urbanas cuyo objetivo era ordenar y disciplinar tanto esos espacios inundables como a sus habitantes (y a sus cuerpos) (Paiva, 1996; Seabra, 2008).

A partir de la década de 1970, el paradigma del ambientalismo es el que fundamenta, con sus discursos asociados a la idea de la sustentabilidad urbano-ambiental, la transformación del espacio urbano, en general, y de las áreas inundables próximas a las ciudades, en particular. Este paradigma reemplaza al higienismo, puesto que el último, con el pasar del tiempo, empieza a tener cada vez menos efecto en sus funciones de ordenamiento $y$ disciplinamiento del uso del espacio urbano, ante los notables procesos de crecimiento demográfico y espacial, ocurridos entre 1940 y 1970; por lo menos para la realidad urbana de varios de los países latinoamericanos que adoptaron el modelo económico de "Industrialización por Sustitución de Importaciones". En esas décadas las ciudades sufrieron cambios significativos, producto de la industrialización y el crecimiento demográfico y espacial, convirtiéndose, algunas de ellas, en enormes metrópolis. Los paradigmas del higienismo y del ambientalismo no llegaron a convivir, porque el primero no pudo soportar las contradicciones que el enorme proceso de urbanización traía consigo (Seabra, 2008).

En los últimos tiempos el ambientalismo es el que moviliza a la sociedad en su relación con la naturaleza, bajo la idea de un retorno a una naturaleza, si es posible, pura. Este paradigma se levanta sobre las consecuencias negativas de la historia humana de la naturaleza que fueron siendo acumuladas 
en un baúl que almacena las "consecuencias no deseadas" de los tiempos históricos (Seabra, 2008.); dentro del cual también se encuentra la transformación y degradación de las condiciones ecológicas de las tierras inundables. El paradigma del ambientalismo, de acuerdo con Seabra (2008), Ilega a la vida cotidiana y a los sujetos en nombre de una racionalidad en el uso del espacio, del tiempo y de las cosas, gracias a las técnicas de información actuales. Esta racionalidad, bajo los preceptos de la sustentabilidad, alcanza a la sociedad y es propuesta en términos prácticos; asimismo, sirve de fundamento para la acción de las empresas y los gobiernos. Es Ilamativo ver como, de un tiempo a esta parte, todas las cosas, relaciones y productos comienzan a ser observados, medidos y avalados, con base en el criterio de la sustentabilidad. Pareciera que se busca la sustentabilidad de todo (Seabra, 2008).

En materia urbana, por ejemplo, se fueron produciendo gran cantidad de propuestas políticas de desarrollo urbano sustentable en los últimos años. Este tipo de discurso ha recibido gran cantidad de críticas. Peter Marcuse (1998), por ejemplo, sostiene que nadie que está interesado en la justicia quiere sustentar las cosas tal cual como están en la actualidad; es decir, la sustentabilidad puede simplemente, en la mayor parte de los casos, sustentar un injusto statu quo y enmascarar los reales conflictos de intereses que están en juego en cuanto a los aspectos que hacen a la producción del espacio, en este caso el vinculado a la urbanización de áreas inundables.

A pesar del poder de lobby que pueden ejercer los cuerpos técnicos a través de sus discursos especializados, coincidimos con Hilda Herzer (2001), quien considera que el monopolio en las alternativas técnicas para solucionar estas problemáticas complejas, como las inundaciones, es virtual. Según esta autora, ese sesgo de tipo ingenieril refleja, en realidad, el ejercicio de poder de otros grupos dentro de la sociedad que conservan fuertes intereses en los lugares en cuestión, entre los que se destacan: propietarios de tierras, rentistas, empresas constructoras, desarrolladores urbanos, developers, inmobiliarias, estudios de arquitectura, entre otros. Esas decisiones, entonces, no son indiferentes frente a los intereses de esos grupos involu- crados, sino que, por lo general, terminan beneficiando a aquellos con mayor poder económico y político dentro de los contextos sociales y urbanos donde se implantan.

\section{Las técnicas "hidráulicas" como valorización urbana de áreas inundables}

Preguntarse por qué se ejecutan obras de infraestructura hidráulica en determinados lugares y no en otros, es decir, cuestionar la ubicación y distribución espacial de esas obras, forma parte de las indagaciones claves (y propiamente geográficas) para lograr entender de manera más profunda los procesos asociados a la producción de espacios de riesgo de desastre desiguales. Estas preguntas nos conducen necesariamente a incursionar en los procesos de valorización del espacio; en este caso, sobre las particularidades que esos procesos adquieren respecto a la urbanización de áreas inundables.

La transformación de las condiciones de sitio en áreas inundables a través de realización de distinto tipo técnicas "hidráulicas" representa una incorporación de valor en esos espacios, a partir de la aplicación de trabajo, técnica y capitales. Con ellas cambian (a veces no totalmente) las condiciones físicas de las áreas implicadas, dado que dejarían de ser inundables o afectables ante los excesos hídricos. Según Lindón (1989), esa modificación en las condiciones de "fertilidad urbana"4, conlleva a un cambio en la valorización social del espacio, el cual termina reproduciendo, a menudo, un cambio en la jerarquización de esas áreas. Ese cambio de valor del espacio repercute en la modificación del uso social y en la apropiación de esas áreas. En varias oportunidades se ha verificado que la realización de obras de infraestructuras hidráulicas influye para que áreas inundables desvalorizadas y ocupadas por grupos de bajos ingresos se transformen en áreas valorizadas y ocupadas (desplazando a los anteriores habitantes) por nuevos grupos con mejores condiciones socioeconómicas y, también, por actividades productivas de capital intensivo.

\footnotetext{
4 A las que define esta autora como aquellas características físicas del espacio para soportar los usos urbanos, entre ellas: la resistencia del suelo, la orientación, la iluminación, la altura o cota, etcétera.
} 
Es por ello que las soluciones hidráulicas en áreas inundables (es decir, a través de la aplicación de técnicas "hidráulicas") en los ámbitos urbanos implican, muchas veces, cambios en la tenencia de la tierra de esas áreas. Estos procesos tienen consecuencias diferenciales sobre los distintos grupos socioeconómicos involucrados; mientras que algunos se benefician ocupando esas nuevas áreas (que podría llamarse protegidas), otros se ven perjudicados; estos últimos deben abandonar esas áreas y terminan por relocalizarse en otros lugares de la ciudad, a menudo, con peores condiciones de fertilidad urbana (más inundables, degradadas ambientalmente, alejadas y peores servidas por la oferta de servicios públicos, por ejemplo) (Clichevsky, 2003).

Estos procesos de reconversión de tierras "anfibias" a tierras "firmes" a partir de la incorporación de valor implicada en las inversiones de infraestructuras públicas o de capital privado, posibilitaron la valorización de las mismas. Así las cosas, las técnicas asociadas con la Modernidad, legitimadas por los discursos higienistas o (más recientemente) ambientalistas, permitieron en distintos momentos históricos, enterrar miasmas y superficies infectadas o recuperarlas sustentablemente y acrecentar así los horizontes de expansión urbana en terrenos que en otro momento no tenían prestigio social, pero mediante intervenciones técnicas patrocinadas por el poder público o el capital privado, han sido transformadas en áreas y vecindades valorizadas (Henrique, 2006).

\section{Las técnicas "hidráulicas" como amplificación de riesgo de desastre}

En varias oportunidades los diques, reIlenos, polders, canalizaciones, entre otras técnicas "hidráulicas", permitieron otorgarles usos productivos o residenciales a distintas áreas inundables incorporadas a los procesos de urbanización. A través de la implementación de este tipo de obras, las áreas inundables terminan siendo valorizadas y ocupadas de manera permanente. Asimismo, el desarrollo de este tipo de técnicas construye, en la percepción de los grupos sociales que quedan dentro de esas áreas defendidas o rellenadas, un falso sentido de seguridad, es por ello que este tipo de obras también se las ha denominado como obras trampa. De tanto en tanto la dinámica natural se encarga de quebrantar esa seguridad a través de desastres, recordándonos que esas tierras que forman parte, por ejemplo, de un valle de inundación, a pesar de los intentos por su dominación técnica, continúan perteneciendo al dominio de las aguas.

Comúnmente ante cada nueva inundación se responde a través de mayor aplicación e inversión en técnicas "hidráulicas", las cuales terminan incentivando una nueva oleada de ocupación de las áreas dañadas. A ello, Acosta (2001) lo ha denominado como el "dilema de la ocupación de las áreas de riesgo", que consiste en un esquema cerrado circular en el que intervienen en forma sucesiva cuatro componentes: inversión en obras, daño, reconstrucción y nueva inversión en obras. Por otro lado, cuando las áreas bajas ocupadas involucran grandes inversiones en bienes y en obras de infraestructuras, la respuesta técnica se ve retroalimentada como la solución más adecuada ante un nuevo acontecimiento; estas soluciones gravitan a menudo en la aplicación de técnicas "hidráulicas" cada vez más imponentes y costosas; así, en este círculo se aumentan los niveles de vulnerabilidad social dado la confiabilidad que se le conceden a esas nuevas obras.

La transformación de áreas inundables, vía la aplicación de técnicas "hidráulicas", para la realización de fines urbanos, ha sido criticada por la Geografía y otras Ciencias Humanas y Sociales que abordan los estudios de riesgo de desastre. Uno de los geógrafos que más ha aportado a este tópico fue el estadounidense Gilbert White, quien trabajó en las décadas de 1930 y 1940 en los primeros organismos técnicos desarrollados para planificar y gestionar los recursos hídricos de ese país, luego de la gran inundación de la cuenca del Mississippi en 1927. Esa experiencia práctica, junto a sus avances en el conocimiento académico, le permitió formular a White la idea de la "paradoja hidráulica". Para este autor las obras hidráulicas no disminuyen frecuentemente el potencial destructor de las aguas, sino que tienden a amplificar los efectos negativos de las inundaciones. Considera, asimismo, que a mayores obras de defensas o mitigación, mayores son los daños que puede causar cuando estas colapsan (Saurí, 2006). 
En contrapartida a los altos niveles de transformación impuestos a la naturaleza, en este caso a naturalezas inundables fuertemente artificializadas por las técnicas "hidráulicas", Ilama la atención las consecuencias impredecibles e inciertas, como así también las desventajas a las que las sociedades deben hacer frente cuando asumen tales transformaciones (Meerganz, 2006). Precisamente, esas consecuencias adversas derivadas del desconocimiento de la respuesta de la naturaleza al intenso nivel de transformación técnica aplicado, conllevan a la generación de nuevos escenarios de riesgo de desastre, cuya base se encuentra, en parte, en la potenciación de los fenómenos peligrosos. Ejemplo de esto último puede ser las transformaciones de cuencas y de ecosistemas inundables (incorporadas en los últimos tiempos dentro de la categoría ecológica de humedales) que derivan en alteraciones de las dinámicas hídricas que rigen esos ambientes anfibios y que terminan constituyendo pérdidas en sus funciones primarias, como pueden ser, entre otras, la de receptáculo de excesos hídricos en tiempos de crecidas, de suma importancia a los fines de los efectos adversos que una inundación puede causar sobre las sociedades.

\section{Consideraciones finales}

Las concepciones fisicalistas o tecnocráticas en torno a los desastres, los riesgos a ellos asociados y las posibles estrategias de mitigación ante estos problemas continúan predominando tanto en el conocimiento académico como en las propuestas de los organismos internacionales especializados en la temática (Hewitt, 1983; Lavell, 1996a; Lavell, 2004). El conocimiento hasta el momento alcanzado por parte de las Ciencias Humanas y Sociales sobre desastres y riesgo de desastre ha procurado enriquecer y superar esas concepciones todavía afianzadas. No obstante ello, han sido escasos los trabajos elaborados por esas disciplinas en los que se logre un marco teórico-conceptual significativo que permita penetrar en las profundas raíces sociales de estos complejos eventos, todavía asociados esencialmente con los fenómenos físico naturales extremos.

Las reflexiones hasta aquí desplegadas procuraron presentar aquellos trabajos supe- radores de las concepciones de los desastres y riesgos de desastre en las que todavía aparece la naturaleza escindida de la sociedad, bajo los conceptos de peligro (o amenaza) y de vulnerabilidad social que, a pesar de ser muchas veces entendidos de manera dinámica, continúan siendo analizados como realidades encerradas en compartimentos estancos, por un lado, la naturaleza y, por otro, la sociedad. Tal como se deriva del recorrido teórico presentado, una de las propuestas superadoras en torno a estos tópicos, desde los abordajes de las Ciencias Humanas y Sociales, especialmente desde la Geografía crítica, radica en ahondar en la dinámica social que interviene en la producción de riesgo de desastre y, más concretamente, de espacio de riesgo de desastre, tomando como referente la urbanización de áreas inundables y su correspondiente mediación técnica.

Como se ha podido advertir, urbanizar tierras inundables requiere de la elaboración de un conjunto de estrategias técnicas, las cuales están mediadas por la forma en que se producen las relaciones sociales y sus vínculos con las condiciones físico-naturales. La incorporación de las condiciones de inundabilidad al proceso de urbanización está (y estuvo) atravesado por las relaciones de poder entre los distintos grupos que se disputan esas tierras inundables para sí, y a través del dominio que los grupos más poderosos tienen sobre esas tierras para mantener su posición, tanto en términos de propiedad privada, de desarrollo técnico y de monopolio de los organismos de gestión en materia hidráulica (Pérez Picazo y Lemeunier, 1990).

Desentrañar el lugar que las técnicas "hidráulicas" han desempeñado a lo largo de la historia de la urbanización de áreas inundables, se convierte en un aspecto esencial en el análisis de los procesos que participan de la producción de espacio de riesgo de desastre para ese tipo de áreas. Es, precisamente, el control de las inundaciones el elemento que prima en la organización y en la producción de esos espacios que se procuran transformarlos en urbanos. Sobre ese control técnico, de acuerdo con Pérez Picazo y Lemeunier (1990), se constituye la base donde se sustenta el poder de las clases dominantes, unas veces, por medio de la transformación 
de tierras inundables en no inundables en las que son utilizadas técnicas de control y/o mitigación y, otras veces, a través del monopolio de los organismos de gestión en materia hidráulica que favorecen a determinados grupos frente a otros. El desarrollo de técnicas "hidráulicas" en tierras inundables por parte de los grupos más poderosos debe comprendérselo como estrategias de utilidad no solo para la valorización urbana de esas áreas, sino también para la dominación política; es precisamente a través de las técnicas -entre otros tantos aspectos- que esos grupos logran mantener su lugar de privilegio, profundizando las desigualdades.

Parafraseando a Swyngedouw (2004), los flujos del dinero, los flujos del poder y en este caso el control técnico sobre los flujos del agua, se encuentran materialmente imbricados en los procesos de urbanización de las tierras inundables. Esto implica que la transformación técnica destinadas a fines urbanos está asentada (más aún a partir del avance del modo de producción capitalista) sobre relaciones socioeconómicas y políticas claramente asimétricas, que terminan, a menudo, reproduciendo desigualdades espaciales, las que también se expresan en términos de riesgo de desastre.

\section{Referencias bibliográficas}

ACOSTA, J. Las inundaciones en Argentina. En: KREIMER, A.; KULLOCK, D. y VALDÉS, J. (eds.). Inundaciones en el Área Metropolitana de Buenos Aires, Washington: Banco Mundial, 2001, p. 17-32.

BAKKER, K. Neoliberalizing Nature? Market Environmentalism in Water Supply in England and Wales. Annals of the Association of American Geographers, 2005, vol. 95, № 3, p. 542-565.

BLAIKIE, P.; CANNON P.; DAVIS, I. y WISNER, B. Vulnerabilidad: el entorno económico, social y político de los desastres. Perú: LA RED/ITGD; Bogotá: Tercer Mundo Editores, 1994. Disponible en Internet: http:// www.desenredando.org

CALDERÓN, G. Construcción y reconstrucción del desastre. México: Plaza y Valdés, 2001.
CLICHEVSKY, N. Urban land markets and disasters: floods in Argentina's cities. In: KREIMER, A. et al. (eds.). Building safer cities: the future of disaster risk. Washington: World Bank, Disaster Risk Management Series, 2003, p. 165-176.

CUTTER, S. The geography of social vulnerability: race, class, and catastrophe. Understanding Katrina: Perspectives from the Social Sciences, 2006. Disponible en Internet: http://understandingkatrina.ssrc.org/Smith/

DAVIS, M. Los Angeles after the storm: the dialectic of ordinary disaster. Antipode, 1995, No 273, p. 221-241.

DAVIS, M. Ecology of fear: Los Angeles and the imagination of disaster. New York: Vintage Books, 1999.

DEL MORAL, L. El agua en la organización del espacio urbano: el caso de Sevilla y el Guadalquivir. Doc. Anàl. Geogr. 1997, No 31, p. 117-127.

GONZÁLEZ, S. Ciudad visible vs. ciudad invisible. Gestión urbana y manejo de inundaciones (Ciudad de Buenos Aires) en la baja cuenca del arroyo Maldonado. Tesis de doctorado. Buenos Aires: Facultad de Filosofía y Letras, Universidad de Buenos Aires, Argentina, mimeo, 2009.

HABERMAS, J. Ciencia y técnica como 'ideología'. Madrid: Tecnos, 1985.

HENRIQUE, W. A cidade e a natureza: a apropiação, a valorização e a sofisticação da natureza nos empreendimentos inmobiliários de alto padrão em São Paulo. GEOUSP, 2006, No 20, p. 65-77.

HERZER, H. Inundaciones en el Área Metropolitana de Buenos Aires: participación de las organizaciones sociales. En: KREIMER, A.; KULLOCK, D. y VALDÉS, J. (eds.). Inundaciones en el Área Metropolitana de Buenos Aires. Washington: Banco Mundial, 2001, p. 166-181.

HERZER, H. y GUREVICH, R. Degradación y desastres. Parecidos y diferentes. En: FERNÁNDEZ, M. A. (comp.). Ciudades en Riesgo. Lima: La Red, 1996. 
HEWITT, K. Interpretations of Calamity. London: Allen and Unwin, 1983.

KELMAN, I. Natural disasters do not exist (natural hazards do not exist either), Ver. 1. Oslo: Ilan Kelman, 2007. Disponible en Internet: http://www.ilankelman.org/ miscellany/NaturalDisasters.rtf

KLEIN, N. Respuesta ante los desastres, solo para los elegidos. Realidad Económica, 2007. Disponible en: http://www.iade.org.ar/ modules/noticias/article.php?storyid=2102

LAVELL, A. Introducción. En: FRANCO, E. y LAVELL, A. (eds.). Estado, sociedad y gestión de los desastres en América Latina. En busca del paradigma perdido. Bogotá: La Red, 1996a, p. 11-32.

LAVELL, A. Degradación ambiental, riesgo y desastre urbano: problemas y conceptos. En: FERNÁNDEZ, M. A. Ciudades en riesgo. Lima: LA RED/USAID, 1996b.

LAVELL, A. La red de estudios sociales en prevención de desastres en América Latina. LA RED: Antecedentes, formación y contribución al desarrollo de los conceptos, estudios y la práctica en el tema de los riesgos y desastres en América Latina: 1980-2004. Ciudad de Panamá: La RED, 2004. Disponible en Internet: http://www. la-red.org/public/varios/2004/LAREDAFCDCEPTRDAM/

LINDÓN, A. Ciudad de Buenos Aires: producción de espacio urbano en sitios vulnerables a inundación entre 15801880. Tesis de Licenciatura. Buenos Aires: Departamento de Geografía, Facultad de Filosofía y Letras, Universidad de Buenos Aires, Argentina, mimeo, 1989.

MANSILLA, E. Notas para una reinterpretación de los desastres. En: MANSILLA, E. (ed.). Desastres: modelo para armar. Lima: LA RED, 1996, p. 58-70.

MANSILLA. E. Riesgo y ciudad. Ciudad de México: Universidad Autónoma de México, División de Estudios de Posgrado, Facultad de Arquitectura, 2000. Disponible en Internet: http://www.desenredando.org
MARANDOLA, E. \& HOGAN, D. Natural Hazards: O estudo geográfico de riscos e perigos. Ambiente e Sociedade, 2004, vol. 7, No 2, p. 95-109.

MARCUSE, P. Sustainability is not enough. Environment and Urbanization, 1998, vol. 10, No2, p. 103-111.

MEERGANZ, G. Flujos de agua, flujos de poder. La aportación de Erik Swyngedouw al debate sobre los recursos hídricos en Latinoamérica y en el Estado español. Doc. Anàl. Geogr., 2006, № 47, p. 129-139.

MEINDL, C.; ALDERMAN, D. \& WAYLEN, $P$. On the importance of the environmental claims-making: the rol of James O. Wright in promoting the drainage of Florida's everglades in the early twentieth century. Annals of the Associations of American Geographers, 2002, vol. 94, No 4, p. 682701.

MITCHELL, J. Natural disasters in the context of mega-cities. In: MITCHELL, J. Crucibles of Hazard: Mega-cities and disasters in transition, UNU, 1999.

NEL-LO, O. y F. MUÑOZ. El proceso de urbanización. En: ROMERO, J. (coord.). Geografía Humana. Procesos, riesgos e incertidumbres en un mundo globalizado. Barcelona: Ariel, 2004, p. 255-332.

O'CONNOR, J. Causas naturales. Ensayos del marxismo ecológico. Ciudad de México: Siglo XXI, 2001.

O'KEEFE, P.; K. WESTGATE \& WISNER, B. Taking the Naturalness out of Natural Disasters. Nature, 1976, № 260, p. 566-567.

ORGANIZACIÓN DE ESTADOS AMERICANOS (OEA). Manual sobre el manejo de peligros naturales en la planificación para el desarrollo regional integrado. Washington: Departamento de Desarrollo Regional y Medio Ambiente de la Organización de Estados Americanos, 1993.

PAIVA, V. Entre miasmas y microbios: la Ciudad de Buenos Aires bajo la lente del higienismo. Buenos Aires 1850-1890. Área, 1996, No 4, p. 23-31. 
PÉREZ PICAZO, M. T. y LEMEUNIER, G. Introducción. En: PÉREZ PICAZO, M. T. y LEMEUNIER, G. (ed.). Agua y modo de producción. Barcelona: Editorial Crítica/ Historia del mundo moderno, 1990, p. 21-53.

ROSWELL, E. Rius i ciutats: amenaces i potencialitats. Doc. Anàl. Geogr., 1997, No 31, p. 23-34.

SANTOS, M. Técnica, espaço e tempo: globalização e meio técnico-científicoinformacional. São Paulo: HUCITEC, 1994.

SANTOS, M. La naturaleza del espacio. Técnica y tiempo. Razón y emoción. Barcelona: Ariel, 2000.

SAURÍ, D. Nota necrológica. Gilbert F. White (Chicago, 1911-Boulder, Colorado, 2006). Doc. Anàl. Geogr., 2006, No 48, p. 13-14.

SEABRA, O. A socialização da natureza: rios e várzeas de São Paulo. São Paulo: DGFFLCH-USP, texto de clase inaugural del año electivo 2008, inédito, 2008.

SILVESTRI, G. Las dos orillas. Obras, proyectos y representaciones en el Río de la Plata. En: BOTHAGARAY, J. M. (comp.). El Río de la Plata como territorio. Buenos Aires:
Ediciones Infinito, FADU, Furban, 2002, p. 533-570.

STEINBERG, T. The secret history of natural disaster. Environmental hazards, 2001, No 3, p. 31-35.

SWYNGEDOUW, E. Modernity and Hibridity: nature, regeneracionismo, and the production of the Spanish waterscape: 18901930. Annals of the Association of American Geographers, 1999, vol. 89, № 3, p. 443465.

SWYNGEDOUW, E. Social Power and the Urbanization of Water. Londres: Oxford University, 2004.

WATTS, M. Hazards and crises: a political economy of drought and famine in northern Nigeria. Antipode, 1983, vol. 15, No 1, p. 24-34

WESTGATE, K. \& O'KEEFE, P. Natural disasters. Bradford: Disaster Research UnitUniversity of Bradford, 1976.

WISNER, B. Is there a geographical theory of terror? In: Los Angeles, Panel Presentation at the Annual Meeting of the Association of American Geographers, 19 to 24 march, 2002. 